\title{
Nosokomiale Pneumonie: Erregerspektrum
}

Santiago Ewig ${ }^{\mathrm{a} *}$ und Sören Gatermann ${ }^{\mathrm{b} *}$

${ }^{a}$ Kliniken für Pneumologie und Infektiologie, Augusta-Kranken-Anstalt Bochum, Thoraxzentrum Ruhrgebiet, Bochum, Deutschland

${ }^{\mathrm{b}}$ Institut für Hygiene und Mikrobiologie, Abteilung für Medizinische Mikrobiologie, Ruhr-Universität Bochum, Bochum, Deutschland

\section{Erregerspektrum}

Das Erregerspektrum der nosokomialen Pneumonie lässt sich nach verschiedenen klinisch relevanten Gesichtspunkten ordnen. Dazu gehören „Häufigkeit“, Vorkommen bei „early onset“ bzw. „late onset“ Pneumonien, „seltene“ und „fragliche Erreger“". Zudem sollten Erreger zusammengefasst werden, die keine Erreger der nosokomialen Pneumonie sind, fälschlicherweise aber häufiger als solche behandelt werden. Eine Übersicht findet sich in Tab. 1.

In diesem Kapitel werden Aspekte der Mikrobiologie, Übertragung, Risikofaktoren, Pathogenese und klinisches Bild der einzelnen Erreger bzw. Erregergruppen dargestellt.

\section{Die wichtigsten Erreger: Staphylococcus aureus und Pseudomonas aeruginosa}

In nahezu allen Übersichten zur Erregerepidemiologie der nosokomialen Pneumonie sind S. aureus und P. aeruginosa die führenden Erreger.

\subsection{Staphylococcus aureus}

\subsubsection{Mikrobiologie}

Staphylokokken sind grampositive Kokken. Sie besiedeln häufig den oberen Respirationstrakt (ca. 30 \%) und die Haut.

Sowohl MSSA (Methicillin-sensible S. aureus) als auch MRSA (Methicillin-resistente S. aureus) spielen eine Rolle als Erreger der nosokomialen Pneumonie. Als MSSA werden Penicillin- und Oxacillin-sensible Stämme von S. aureus bezeichnet; bei ihnen ist die Empfindlichkeit gegenüber ß-Laktamen grundsätzlich erhalten. Als MRSA werden dagegen Stämme bezeichnet, die gegen Oxacillin und damit gegen fast alle B-Laktame unempfindlich sind. Bei der nosokomialen Pneumonie spielen die klassischen Oxacillin-resistenten HA (Hospital-acquired)-MRSA die größere Rolle, CA (communityacquired)- sowie LA (lifestock-associated)-MRSA werden nur selten angetroffen. VISA und VRSA (Vancomycin intermediärresistente bzw. resistente Stämme) sind in Europa selten und spielen bei der nosokomialen Pneumonie (noch) keine Rolle.

MSSA kommt überwiegend (aber keineswegs ausschließlich) im Rahmen der „,early onset“ Pneumonie vor, MRSA in der Regel bei „late onset“ Pneumonie.

\footnotetext{
*E-Mail: sewig@outlook.de

*E-Mail: soeren.gatermann@rub.de
} 
Tab. 1 Erreger der nosokomialen Pneumonie, dargestellt nach verschiedenen Ordnungsprinzipien

\begin{tabular}{|c|c|}
\hline Vorkommen & Erreger \\
\hline Häufigste Erreger & $\begin{array}{l}\text { S. aureus (MSSA und MRSA) } \\
\text { P. aeruginosa }\end{array}$ \\
\hline Häufigste Erreger der „,early onset“ Pneumonie & $\begin{array}{l}\text { S. aureus (MSSA) } \\
\text { Haemophilus influenzae } \\
\text { Streptococcus pneumoniae } \\
\text { E. coli }\end{array}$ \\
\hline Häufigste Erreger der „,late onset“ Pneumonie & $\begin{array}{l}\text { S. aureus (MSSA und MRSA) } \\
\text { Enterobakterien, häufig multiresistent } \\
\text { - E. coli } \\
\text { - Klebsiella pneumoniae/oxytoca } \\
\text { - Enterobacter cloacae/aerogenes } \\
\text { - Serratia marcescens/liquefaciens } \\
\text { - Proteus mirabilis/vulgaris } \\
\text { Nonfermenter } \\
\text { - P. aeruginosa } \\
\text { - Acinetobacter baumannii } \\
\text { - Stenotrophomonas maltophilia }\end{array}$ \\
\hline Seltene Erreger & $\begin{array}{l}\text { Aspergillus spp. } \\
\text { Legionella spp. }\end{array}$ \\
\hline Fragliche Erreger & $\begin{array}{l}\text { Anaerobier (gesichert bei Empyemen) } \\
\text { Viren (Herpes simplex und Zytomegalie) }\end{array}$ \\
\hline Keine Erreger & $\begin{array}{l}\text { Koagulasenegative Staphylokokken } \\
\text { Streptokokken (andere als S. pneumoniae) } \\
\text { Neisseria spp. } \\
\text { Corynebacterium spp. } \\
\text { Enterococcus spp. (auch nicht VRE) } \\
\text { Candida spp. }\end{array}$ \\
\hline
\end{tabular}

Cave Der einzige Unterschied zwischen den beiden Formen MSSA und MRSA ist die Empfindlichkeit gegenüber Oxacillin bzw. Methicillin (und weiteren antimikrobiellen Substanzen). Es bestehen keine weiteren Unterschiede, etwa in Übertragung und Pathogenität.

\subsection{2 Übertragung}

Eine Übertragung ist möglich von Mensch zu Mensch durch direkten Kontakt und über Aerosole. Über die Aspiration von oropharyngealem Sekret gelangt der Erreger in die tiefen Atemwege. Selten finden sich Pneumonien, die über eine Bakteriämie entstanden sind (dabei häufig aus einer anderen Infektionsquelle).

\subsubsection{Risikofaktoren}

Die wichtigsten Risikofaktoren für eine MSSA-Pneumonie sind eine Bewusstseinstrübung bzw. ein Trauma (besonders zerebral); klinisch wird der Erreger entsprechend häufig bei der „early onset“ Pneumonie gefunden.

Entsprechend ist der wichtigste Risikofaktor für eine MRSA-Pneumonie eine prolongierte Hospitalisation bzw. Intensivstations-Behandlung sowie antimikrobielle Therapie. MRSA wird somit eher bei ,late

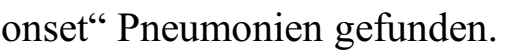

$\mathrm{Ob}$ eine vorbestehende ausschließliche nasale Kolonisation mit MRSA einen Prädiktor für eine MRSA-Pneumonie darstellt, ist nicht eindeutig geklärt (Robiczek et al. 2008; Sarikonda et al. 2010; Dangerfield et al. 2014). Es ist jedoch wahrscheinlich, dass eine tracheobronchiale Kolonisation einen 
solchen Risikofaktor ausmacht. Dasselbe gilt für eine MSSA-Pneumonie (Bergmanns et al. 1996; Rocha et al. 2013; Jang et al. 2014).

\subsubsection{Pathogenese}

Nach Adhärenz in den oberen Atemwegen mit nachfolgender Kolonisation können S. aureus in die unteren Atemwege deszendieren und zu einer Pneumonie führen.

Eine Fülle von Virulenzfaktoren tragen zur Pathogenität von S. aureus bei. Dazu gehören unter vielen anderen die Polysaccharidkapsel, Zellwandbestandteile wie Protein A, der Fibrinogenrezeptor oder das Fibronektin-bindende Protein, daneben viele Exotoxine sowie eine Reihe von Enzymen. Ein große Menge $\alpha$-Toxin scheint die Entwicklung einer nosokomialen Pneumonie zu erleichtern (Stulik et al. 2014). Je nach Virulenz und Immunitätslage bleibt diese bronchopneumonisch oder weitet sich zu einer alveolären Pneumonie aus, ggf. bis hin zur Abszessbildung.

\subsubsection{Klinisches Bild einer Staphylococcus-aureus-Pneumonie}

Ein charakteristisches Krankheitsbild ist nicht bekannt. Allerdings besteht eine Neigung zur Einschmelzung mit Nekrotisierung, Abszessen, Empyemen, Pneumatocelen und Pneumothoraces.

Die Prognose der MSSA-Pneumonie im Rahmen der ,early onset“ Pneumonie ist in der Regel günstig, möglicherweise weil diese lediglich über eine hohe Inokulationsdosis entstanden ist und weniger in der Pathogenität des Erregers begründet liegt. MRSA-Pneumonien weisen eine krude Exzess-Letalität auf, die jedoch nach Adjustierung für Alter und Komorbidität nicht sicher fortbesteht (Combes et al. 2004).

\subsection{Pseudomonas aeruginosa}

\subsubsection{Mikrobiologie}

P. aeruginosa ist ein gramnegatives Stäbchenbakterium, das keine Kohlenhydrate unter anaeroben Bedingungen metabolisieren kann (daher Nonfermenter). Seine Habitate sind Wasser, aber auch Pflanzen und feuchte Böden. P. aeruginosa kolonisiert bei gesunden Personen gelegentlich den Darm, an anderen Orten stellt er beim Gesunden transitorische Flora dar.

Pseudomonas aeruginosa zeichnet sich durch seine ausgeprägte Fähigkeit zur Resistenzentwicklung gegen antimikrobielle Substanzen aus. Beteiligt sind u. a. chromosomal kodierte induzierbare AmpC-ß-Laktamasen, Verluste von Porinen, was die Penetration von Antibiotika in die Zelle behindert, sowie ein erhöhter Efflux von antimikrobiellen Substanzen. Zudem kommen praktisch alle plasmidkodierten Resistenzmechanismen vor. Zunehmend werden auch Carbapenemasen gesehen.

Besonders schwer zu behandeln sind mukoide Stämme, die typischerweise bei der cystischen Fibrose, aber auch bei schwerer COPD bzw. non-CF-Bronchiektasen und ,late onset“ Pneumonien als Kolonisation auftreten. Diese Stämme produzieren die extrazelluläre Substanz Alginat, welche als Matrix eines Biofilms dient. Die besiedelnden Stämme beherbergen mehrere Klone mit unterschiedlichen Resistenzund Virulenzeigenschaften, die durch antimikrobielle Therapie vermindert bzw. umgekehrt selektiert werden können; ein Resistogramm gibt hier keine verlässliche Auskunft mehr darüber, welche Substanzen therapeutisch wirksam sind.

Mukoide Stämme bei nosokomialer Pneumonie zeigen somit je nach erhaltener antimikrobieller Therapie wechselnde Resistogramme, in denen typischerweise (mindestens) die zuletzt gegebene Substanz resistent erscheint. 


\subsection{2 Übertragung}

Zunächst kommt es zu einer Kolonisation der unteren Atemwege. Risikofaktoren dafür sind eine chirurgische Notfallintervention, Alkoholismus, die Gabe nicht anti-pseudomonal wirksamer antimikrobieller Substanzen sowie eine prolongierte Hospitalisation (Talon et al. 1998).

Der wichtigste Übertragungsmechanismus ist die Aspiration oropharyngealen Sekrets. Seltener kann auch eine Inhalation Pseudomonas-kontaminierter Aerosole zugrunde liegen. Ebenso selten kann eine Bakteriämie aus einem anderen Infektionsherd eine Pneumonie zur Folge haben.

\subsubsection{Pathogenese}

P. aeruginosa ist ein opportunistischer Erreger par excellance und verursacht Infektionen typischerweise nur bei schwergradig morbiden Patienten. Es wird daher überwiegend als nosokomialer Infektionserreger angetroffen.

P. aeruginosa weist eine Fülle von Virulenzfaktoren und -mechanismen auf, unter diesen Alginat (verhindert Phagozytose), Lipopolysaccharide, Pili, Geißeln, Exotoxine, Proteasen, Oxidantien sowie das Quorum sensing (Mengenregelung über chemische Transmitter, Biofilmbildung). Diese Ausstattung erklärt zum Teil die Neigung zur nekrotisierenden Pneumonie.

\subsubsection{Risikofaktoren}

Risikofaktoren für eine nosokomiale Pneumonie durch P. aeruginosa umfassen eine tracheobronchiale Kolonisation durch P. aeruginosa, eine schwere pulmonale Komorbidität (COPD und/oder Bronchiektasen) sowie eine ,late onset“" Pneumonie, im Einzelnen eine prolongierte Hospitalisation bzw. Intensivstationsbehandlung bzw. invasive Beatmung sowie eine prolongierte antimikrobielle Therapie (Celis et al. 1988; Rello et al. 1994).

\subsubsection{Klinisches Bild einer Pseudomonas-Pneumonie}

Das wesentliche Charakteristikum der P. aeruginosa-Pneumonie besteht darin, dass diese häufig bei schwerkranken Patienten auftritt, einen schweren Verlauf nimmt (mit Schock und Multiorganversagen), sich nicht selten mit Nekrotisierungen und Einschmelzungen präsentiert, mit einer entsprechend hohen Letalität einhergeht und (nach vorläufiger Beherrschung) aufgrund nicht immer gelingender Eradikation eine Rezidivneigung aufweist (Crouch Brewer et al. 1996; Rello et al. 1998). Letzteres ist Ausdruck der Tatsache, dass häufig keine Eradikation des Erregers gelingt. Die Letalität ist entsprechend hoch und beträgt bis knapp $70 \%$; in jedem Fall liegt sie höher als die nach APACHE II prädizierte Letalitätsrate (Crouch Brewer et al. 1996).

\section{Ein typischer Erreger der ,early onset" Pneumonie: Haemophilus influenzae}

\subsection{Mikrobiologie}

Haemophilus influenzae ist ein kleines pleomorphes, gramnegatives Bakterium. Das Wachstum in der Kultur benötigt zwei Faktoren, den X- und V-Faktor. Seine Abhängigkeit von X (Häm) begründet seinen Namen als „Blutliebhaber“. Es gibt bekapselte und nicht-bekapselte Stämme. Die bekapselten Stämme (mit sechs bekannten Serotypen a-f) verursachen invasive Infektionen, die nicht-bekapselten Infektionen der oberen und unteren Atemwege (diese sind der häufigste Kolonisationskeim bei COPD und auch bei akuten Exazerbationen der am häufigsten nachgewiesene bakterielle Erreger). Auch die unbekapselten Stämme können gelegentlich in der Blutkultur gefunden werden. 
Die Resistenz gegenüber ß-Laktamen wird meist über ß-Laktamasen vermittelt. Andere Resistenzen sind selten, nehmen aber zu (z. B. ß-Laktamase negative Ampicillin- bzw. $\beta$-Laktam-Resistenz (BLNAR bzw. BLNBR) oder ß-Laktamase positive Ampicillin-Clavulansäure Resistenz (BLPACR)).

\section{2 Übertragung}

Haemophilus influenzae haben ihr Habitat ausschließlich in den Atemwegen der Menschen. Nichtbekapselte Stämme kolonisieren den Atemwegstrakt in 30 bis $80 \%$ der untersuchten Fälle, bekapselte Stämme (vor allem Typ b) in 2 bis $4 \%$ der ungeimpften Population. Allerdings wechseln die kolonisierenden Stämme nach Tagen bis Monaten. Die Übertragung geschieht über Tröpfcheninfektion oder kontaminiertes Atemwegsmaterial.

\subsection{Pathogenese}

Die Entwicklung einer Pneumonie setzt eine Aspiration von Sekreten mit hohen Keimzahlen sowie einen immunologisch lokal (bronchial) oder systemisch geschwächten Wirt voraus. Die Immunität ist bei bekapselten Stämmen kapsel-, bei nicht-bekapselten stammspezifisch.

\subsection{Risikofaktoren}

Risikofaktoren umfassen inhalatives Zigarettenrauchen und COPD. Auch bei Konstellationen, die eine Aspiration begünstigen (z. B. Alkoholismus, ZNS- und Ösophaguserkrankungen mit Schluckstörungen) tritt Haemophilus influenzae gehäuft auf.

Typischerweise wird H. influenzae bei der ,early onset“ Pneumonie gefunden. Analog S. aureus (in der Regel MSSA) gelangt dieser Erreger in hoher Keimzahl durch die Intubation oder begünstigt durch eine andere, das Bewusstsein beeinträchtigende Kondition in die tiefen Atemwege. H. influenzae tritt gehäuft zusammen mit S. aureus (MSSA) auf.

\subsection{Klinisches Bild der Haemophilus-influenzae-Pneumonie}

Dieses entspricht meist dem einer Bronchopneumonie. Schwere Verläufe sind selten. Die Prognose ist eher gut.

\section{Enterobakterien - E. coli, Klebsiella spp., Enterobacter spp., Serratia marcescens, Proteus spp.}

Enterobakterien gelangen über eine oropharyngeale Kolonisation in die unteren Atemwege und können so zu Pneumonien führen. Die gastrische Kolonisation mit Aufstieg bis in den Oropharyngealtrakt spielt dabei eine wichtige Rolle. Übertragung, Risikofaktoren und Pathogenese sind für diese Gruppe identisch. Das klinische Bild ist uncharakteristisch.

\subsection{Escherichia coli}

\subsubsection{Mikrobiologie}

E. coli sind gramnegative Stäbchen. Virulenzfaktoren sind vielfältig, unter anderem Adhäsine (wie Fimbrien und Pili), Toxine (z. B. Hämolysine), Lipopolysaccharide (LPS) und Kapselbildung.

Resistenzen werden hauptsächlich vermittelt über durch Plasmide erworbene Enzyme, die z. B. für ß-Laktamasen, aber auch ESBL sowie Carbapenemasen kodieren. 


\subsubsection{Klinisches Bild der E. coli-Pneumonien}

Meistens handelt es sich um Bronchopneumonien. Ebenso wie bei Klebsiellen (s. u.) besteht eine Neigung zur Empyembildung. Ein Nachweis von E. coli in der Blutkultur ist noch nicht gleichbedeutend mit einem Erregernachweis der Pneumonie; vielmehr muss zusätzlich vor allem nach Harnwegsinfektionen und abdominellen Infektionen gefahndet werden.

\subsection{Klebsiella spp. (pneumoniae/oxytoca)}

\subsubsection{Mikrobiologie}

Klebsiella spp. sind gramnegative, bekapselte Stäbchen. Sie kommen in der oropharyngealen Flora in bis zu ca. $5 \%$ vor. Die Polysaccharid-Kapsel mit über 70 antigenen Varianten erzeugt die mukoiden Kolonien auf der Agar-Platte und gilt als wichtigster Virulenzfaktor. Dieser wird über die Hemmung der Phagozytose wirksam.

Neben K. pneumoniae sind als Erreger der nosokomialen Pneumonie auch K. oxytoca, seltener andere relevant.

Alle Klebsiellen sind über eine chromosomal kodierte $\beta$-Laktamase natürlich resistent gegen Ampicillin. Eine Multiresistenz wird bei Klebsiellen häufig über Plasmide übertragen, die auch eine Resistenz vom ESBL-Typ vermitteln. Neuerdings muss zusätzlich mit Carbapenemasen gerechnet werden.

\subsubsection{Klinisches Bild der Klebsiellen-Pneumonie}

Im Gegensatz zu ambulant erworbenen Pneumonien mit K. pneumoniae als Erreger verlaufen die nosokomialen Klebsiellen-Pneumonien meist weniger schwer.

\subsection{Enterobacter spp. (cloacae/aerogenes)}

\subsubsection{Mikrobiologie}

Enterobacter cloacae und Enterobacter aerogenes sind die beiden wichtigsten Erreger. Sie weisen als wichtigsten Pathogenitätsfaktor eine Kapsel auf, die sie gegen Phagozytose schützt.

Alle Enterobacter-Spezies besitzen eine chromosomal kodierte AmpC- $\beta$-Laktamase, die induzierbar oder konstitutiv exprimiert werden kann. Stämme mit induzierbar exprimierter AmpC-ß-Lactamase erscheinen in der Testung gegen Piperacillin und Drittgenerations-Cephalosporine sensibel, es werden unter Therapie mit diesen Substanzen aber Mutanten selektiert, die die ß-Laktamase induziert oder konstitutiv exprimieren; diese sind gegen alle $\beta$-Laktame außer Carbapeneme resistent.

\subsection{Serratia marcescens}

\subsubsection{Mikrobiologie}

Serratia marcescens wird häufig in der Umwelt gefunden, seltener im Stuhl; die meisten Übertragungen sind daher exogen. Viele Stämme bilden ein rotes Pigment namens Prodigiosin auf Oberflächen bzw. in der Kultur. Der Erreger ist sehr umweltresistent und kommt daher häufiger bei Ausbrüchen vor.

Serratia marcescens kolonisiert bevorzugt die Atemwege (neben dem Harnwegstrakt). Der Erreger zeichnet sich durch eine erhebliche Umweltresistenz aus. Serratia ist immer resistent gegen Ampicillin aufgrund einer induzierbaren AmpC-ß-Laktamase. Viele Stämme haben Plasmid-kodierte Resistenzen gegen $\beta$-Laktame und Aminoglykoside.

Selten wird Serratia liquefaciens gefunden. 


\subsection{Proteus spp. (mirabilis/vulgaris)}

Mikrobiologie Namengebend ist eine Gestalt in Homers Odysseus, die ihre Form verändert kann. Proteus mirabilis und Proteus vulgaris zeigen eine charakteristische „Swarming motility“ auf der AgarPlatte.

\subsection{Andere}

Andere gramnegative Erreger wie Citrobacter spp., Hafnia alvei, Providencia spp. und Morganella morganii sind nur ausnahmsweise Erreger einer Pneumonie, vielmehr meist Kolonisationserreger.

\section{Nonfermenter (andere als P. aeruginosa): Acinetobacter, Stenotrophomonas}

Bei diesen Erregern handelt es sich um klassische bakterielle opportunistische Infektionen. Sie sind meist Kolonisations- und nur selten Pneumonie-Erreger. Nur wenige antimikrobielle Substanzen sind wirksam, nicht selten besteht eine Carbapenem-Resistenz.

\subsection{Acinetobacter baumannii}

\subsubsection{Mikrobiologie}

Potenziell pathogen sind Isolate der Acinetobacter baumanii-Gruppe (im angelsächsischen Sprachraum gelegentlich auch A. baumannii-A. calcoaceticus-Complex), zu der u. a. A. baumannii, A. pittii und A. nosocomialis gehören, deren Unterscheidung allerdings eine Herausforderung darstellen kann. Dagegen sind andere Acinetobacter spp. nur sehr selten Infektionserreger. Es handelt sich um gramnegative Bakterien, die in der stationären Phase als Diplokokken erscheinen, in der Wachstumsphase haben sie Stäbchenform.

Acinetobacter haben ihr Habitat ubiquitär auf Böden und im Wasser, in Nahrung und in Abwässern. Auf unbelebten Oberflächen können sie sehr lange überleben, von dort aus können Übertragungen erfolgen. Sie können auch Haut und Schleimhäute besiedeln, auch bei Gesunden; besiedelnde Isolate gehören allerdings nur selten zu der pathogenen A. baumannii-Gruppe.

Acinetobacter spp. sind gefürchtet aufgrund ihrer langen Persistenz in der Umgebung sowie ihrer multiplen Resistenz gegen wichtige antimikrobielle Substanzen. Letztere resultiert aus der Expression von B-Laktamasen, alterierten Porinkanälen und Effluxpumpen.

\subsection{2 Übertragung}

Die Übertragung erfolgt von Mensch zu Mensch oder über Oberflächen bzw. Instrumente.

\subsubsection{Pathogenese}

Virluenzfaktoren sind eine Polysaccharid-Kapsel sowie die Fimbrien, die eine Adhärenz auf den Epithelzellen begünstigen. Ansonsten weist Acientobacter keine hohe Pathogenität auf.

\subsubsection{Risikofaktoren}

Diese umfassen eine schwere Grunderkrankung, eine schwere akute Erkrankung, eine prolongierte Hospitalisation bzw. intensivstationäre Therapie bzw. invasive Beatmung, eine Tracheostomie sowie eine prolongierte antimikrobielle Therapie (Fagon et al. 1996). 


\subsubsection{Klinisches Bild der Acinetobacter Pneumonie}

Die Klinik der Acinetobacter-Pneumonie ist unspezifisch. Häufiger besteht eine multilobäre Pneumonie. Die Letalität ist sehr hoch und erreicht 70 \%; die Prognose bessert sich, wenn eine adäquate antimikrobielle Therapie über drei Tage bestanden hat. Offenbar besteht eine Exzess-Letalität von $25 \%$ (Lortholary et al. 1995).

\subsection{Stenotrophomonas maltophilia}

\subsubsection{Mikrobiologie}

Stenotrophomonas ist ein gramnegatives Bakterium, ebenfalls ein Nonfermenter. Es kommt vor allem in Wasser und feuchter Umgebung vor. Stenotrophomonas ist nur auf wenige Substanzen empfindlich (Cotrimoxazol, gelegentlich Tigecyclin), sodass sie im Krankenhaus selektiert werden. Entsprechend finden sich gehäuft Isolate von S. maltophilia auf Intensivstationen, die häufig Carbapeneme, aber auch Cephalosporine und Fluorchinolone einsetzen.

Grenzwerte der Empfindlichkeit sind nicht definiert; es gibt keinen klaren Zusammenhang von Resistenz- und Therapieergebnis. Grundsätzlich ist die Virulenz sehr gering, und es bleibt häufig unsicher, ob Stenotrophomonas tatsächlich der zugrundeliegende Erreger ist.

\subsection{2 Übertragung}

Die Übertragungswege (Mensch zu Mensch oder Umgebung bzw. Instrumente zu Patient) sind divers bzw. noch nicht eindeutig geklärt (Gherardi et al. 2005). Die hohe genetische Variabilität der meisten Stämme spricht dafür, dass das Bakterium meist von verschiedenen Quellen stammt, häufig wohl auch außerhalb des Krankenhauses erworben wird und erst durch (wiederholte) antimikrobielle Therapie selektiert wird.

\subsubsection{Pathogenese}

Stenotrophomonas hat kaum bekannte Virulenzfaktoren. Das Bakterium kann Biofilme bilden (über den „diffuse signaling factor“ (DSF)), zudem kann die Kolonisation durch das Lipopolysaccarid (LPS) der äußeren Membran ermöglicht werden. Proteasen, Lipasen und andere extrazelluläre Enzyme können eine Rolle spielen.

Der Erreger spielt möglicherweise eine wichtige Rolle als Kofaktor schwerer Pneumonien durch gramnegative Erreger. Interessanterweise konnte der Erreger experimentell durch Ausscheidung von induzierbaren $\beta$-Laktamasen das Wachstum von Serratia marcescens und P. aeruginosa selbst unter Therapie mit Imipenem und Ceftazidim unterhalten (Kataoke et al. 2003).

\subsubsection{Risikofaktoren}

Diese entsprechen denen der Acinetobacter-Pneumonie. Insbesondere eine wiederholte bzw. prolongierte antimikrobielle Therapie mit breitem antimikrobiellem Spektrum (Carbapeneme, Cephalosporine, Fluorchinolone) selektiert Stenotrophomonas. Zudem wurden eine schlechte Funktionalität und eine COPD als Risikofaktoren identifiziert.

\subsubsection{Klinisches Bild der S. maltophilia-Pneumonie}

Die Mehrzahl der Isolate von Stenotrophomonas maltophilia entsprechen einer Kolonisation. Die Klinik der S. maltophilia-Pneumonie ist unspezifisch. 


\section{Legionella spp.}

Nosokomiale Pneumonien durch Legionella spp. können im Rahmen von Ausbrüchen vorkommen. Der Legionellen-Antigentest identifiziert im Wesentlichen nur L. pneumophila Serogruppe 1, die ca. 90 \% der Legionellosen ausmachen (Boccia et al. 2006).

Im Falle einer Häufung von Pneumonien müssen daher zusätzlich respiratorische Sekrete auf Legionellen kulturell sowie durch PCR untersucht werden.

Jeder Fall einer nosokomialen Legionellose muss Anlass zu einer Überprüfung des Wasserversorgungssystems eines Krankenhauses sein (Bartley et al. 2006).

\section{Anaerobier}

Mikrobiologie Unter Anaerobiern werden Bakterien zusammengefasst, die nur in Abwesenheit von Sauerstoff wachsen können oder wenigstens auf deutlich reduzierte Konzentrationen des Gases angewiesen sind. Sie können grampositiv oder gramnegativ, Kokken oder Stäbchen sein.

Studien aus den 70er-Jahren, denen eine transtracheale Aspirationstechnik zur Gewinnung respiratorischer Sekrete zugrunde lag, haben Anaerobier als häufige Erreger der nosokomialen Pneumonie und der Asprationspneumoniae beschrieben. Spätere Untersuchungen mit bronchoskopisch gewonnenen Materialien haben widersprüchliche Ergebnisse erbracht (Doré et al. 1996; Robert et al. 1999; Marik und Careau 1999). Eine tracheobronchiale Kolonisation scheint häufig vorzuliegen (Robert et al. 2003).

Sicher ist, dass der Nachweis von Anaerobiern aus respiratorischen Sekreten technisch schwierig und in vielerlei Hinsicht fehleranfällig ist. Zudem erweist sich die Identifikation eines Anaerobiers als Erreger meist als fraglich. Untersuchungen zum Thema sind entsprechend spärlich geworden. Vor diesem Hintergrund wird heute in der Regel gar nicht speziell nach Anaerobiern gesucht.

Bei Pleuropneumonien mit Empyem allerdings werden Anaerobier gehäuft isoliert und können als typische Erreger angesehen werden.

\section{Pilze: Candida spp., Aspergillus spp.}

\subsection{Candida spp.}

Nosokomiale Pneumonien durch Candida spp. beim nicht schwergradig immunsupprimierten Wirt (zur Definition siehe Kap. > Definitionen) gibt es nicht! Dies kann nicht deutlich genug herausgestrichen werden, da diese häufig bei kritischen Patienten als einziges Isolat identifiziert und daher (meist trotz besseren Wissens) behandelt werden.

Candida spp. sind in respiratorischen Sekreten häufig nachweisbar nach antimikrobieller Breitspektrum-Therapie.

Untersuchungen zum Thema haben jedoch weder im klinischen Setting (Rello et al. 1998; Terraneo et al. 2015) noch autoptisch (el-Ebiary et al. 1997; Meereseman et al. 2009) eindeutige Belege für nosokomiale Candida-Pneumonien gefunden. Übereinstimmend berichten die Autoren von einer hohen Inzidenz von Kulturen von Sekreten der tiefen Atemwege mit Candida spp., jedoch konnte kein überzeugender histologischer Nachweis einer einzigen Candida-Pneumonie geführt werden. Candida spp. wurde besonders bei schweren Verläufen gefunden und häufig behandelt, die antifungale Therapie hatte jedoch keinen Einfluss auf den Ausgang (Terraneo et al. 2015).

Es gibt nur eine einzige ältere autoptische Studie von onkologischen Patienten, die CandidaPneumonien histologisch belegt hat (Haron et al. 1993). Demnach entsteht diese im finalen Stadium im 
Rahmen einer Deszension aus dem Oropharyngealtrakt in die tiefen Atemwege, nur ausnahmsweise hämatogen. Solche Verläufe dürften jedoch unter heutigen Bedingungen aufgrund aktueller Supportivkonzepte in der Onkologie, die eine Behandlung einer oralen und ösophagealen Candidiasis einschließen, kaum oder gar nicht mehr vorkommen.

Candida spp. können nekrotisches Gewebe sekundär kolonisieren. Auch daraus kann jedoch keine Behandlungsindikation abgeleitet werden.

Interessant ist allerdings der Befund einer positiven Assoziation von Candida spp. mit der Ausbildung einer P. aeruginosa-Pneumonie (Azoulay et al. 2006) bzw. MDR-Erregern und einer erhöhten Letalität (Hamet et al. 2012). Ebenso konnten Hinweise auf eine geringere Rate von Kolonisation und Pneumonie durch P. aeruginosa durch eine Candida-wirksame antifungale Therapie gefunden werden (Nseir et al. 2007). Solange jedoch keine Daten vorliegen, die einen eindeutigen Vorteil einer Behandlung von Candida spp. belegen, darf daraus jedoch keine Indikation für eine Therapie der Candida-Kolonisation abgeleitet werden.

\subsection{Aspergillus spp.}

Aspergillus spp. können ausnahmsweise Erreger nosokomialer Pneumonien auch bei definitionsgemäß nicht schwergradig immunsupprimierten Patienten sein. Allerdings stehen die Grunderkrankungen für Dispositionen sozusagen im Graubereich zur schweren Immunsuppression (z. B. schwere COPD, Leberzirrhose, Autoimmunerkrankungen).

\subsubsection{Mikrobiologie}

Aspergillus Sporen kommen ubiquitär vor, finden sich jedoch besonders häufig in Pflanzenböden. Infektiös sind die Konidien. Unter günstigen Bedingungen kommt es zur Keimung und Hyphenbildung. Hyphen sind charakterisiert durch Abzweigungen im 45 Grad Winkel. Ein Hyphengeflecht wird als Myzel bezeichnet. Konidiophoren an der Spitze des Geflechts bilden neue Sporen aus.

Es handelt sich meist um Aspergillus fumigatus, selten sind andere Spezies wie A. niger, A. flavus oder A. terreus.

\subsection{2 Übertragung}

Aspergillus-Sporen werden aerogen (in der Konidien-Form) übertragen.

\subsubsection{Pathogenese}

Immungesunde können in die Alveolen gelangte Konidien problemlos eliminieren. Unter günstigen Bedingungen kommt es jedoch zur Invasion. Dabei wird eine Fülle von Genen aktiviert, die evolutionär ausgebildet das Überleben in verschiedenen Umgebungen gesichert haben. Diese regulieren die Zellwandzusammensetzung, das Hyphenwachstum, die Aquisition von Nahrungsbestandteilen und Mechanismen der Abwehr ungünstiger Faktoren wie des oxidativen Stresses. Aspergillus spp. produzieren eine Reihe von Mykotoxinen, die allerdings für die Pneumonie keine bekannte Bedeutung haben (Hohl und Feldmesser 2007).

\subsubsection{Risikofaktoren}

Konditionen, die ein Risiko für Aspergillosen bergen, umfassen die schwere COPD (regelhaft mit systemischer Steroidtherapie, fortgesetzt oder wiederholt in der Vergangenheit), Autoimmunerkankungen (meist unter immunsuppressiver Medikation), Leberzirrhose sowie Patienten mit der Anamnese einer soliden Organtransplantation (Meersseman et al. 2004; Vandewoude et al. 2006). 


\subsubsection{Klinisches Bild der Aspergillus-Pneumonie}

Die klinische Präsentation der Aspergillus-Pneumonie ist unspezifisch. Gelegentlich sind Hämoptysen bzw. eine Hämoptoe auffällig. Wegweisend ist eher der radiologische Befund mit Knötchen, Halos, Einschmelzungen, Kavernenbildungen und dem Sichel-Zeichen (,air-crescent sign“). Charakteristisch sind auch die Ausbreitung entlang des bronchovaskulären Bündels sowie periphere, trianguläre, infarktoide Konsolidierungen. Bronchoskopisch werden mitunter tracheobronchiale Pseudomembranen gefunden (Ewig et al. 2011; Taccone et al. 2015).

Die Prognose ist sehr schlecht ( $>80 \%$ Letalität); sie steht in Abhängigkeit vom Zeitpunkt der Diagnose, worauf sich die Forderung nach einer möglichst frühen Erkennung und Einleitung einer Aspergillus-wirksamen Therapie ergibt (Meersseman et al. 2004).

\section{Viren}

Viren sind erst kürzlich als Erreger nosokomialer Pneumonien, in erster Linie bei VAP, anerkannt worden. Ihre exakte Rolle bei VAP ist jedoch noch nicht definiert. Dabei spielen zurzeit nur zwei Herpesviren eine Rolle: Herpes simplex Virus 1 (HSV-1) und Zytomegalievirus (CMV).

\subsection{Mikrobiologie}

Ein Nachweis eines der Herpesviren allein belegt noch keine Pneumonie; dieser kann entweder durch eine Kontamination durch Sekrete erfolgen oder durch eine ausschließliche Ausscheidung ohne Infektion. Hierfür bedarf es vielmehr neben einer positiven Kultur bzw. PCR des zusätzlichen Nachweises eines spezifischen zytopathischen Effekts in Zellen aus der BALF oder in der Histologie. Typischerweise ist der zytopathische Effekt bei HSV-1 charakterisiert durch nukleäre, bei CMV durch zytoplasmatische Einschlüsse.

Auch eine hohe Viruslast kann auf eine Pneumonie hinweisen, allerdings ist noch kein Schwellenwert definiert bzw. validiert worden.

\section{2 Übertragung}

Diese findet im Rahmen der nosokomialen Pneumonie offenbar nicht statt, vielmehr handelt es sich um eine Reaktivierung.

\subsection{Pathogenese}

Herpesviren können im Rahmen schwerer Infektionen reaktiviert werden. Offenbar ist bei Beatmungspatienten mit Schock die Zeit der sekundären Immunparalyse eine Prädisposition. Wahrscheinlich beginnt die HSV-1-Infektion mit einer viralen Reaktivierung in der Mundschleimhaut und setzt sich dann über die Atemwege fort. Aber auch andere Ausbreitungswege (z. B. hämatogen) sind möglich.

\subsection{Risikofaktoren}

Diese sind noch nicht definiert. Betroffene Patienten weisen jedoch häufig eine schwere Infektion bzw. ein ARDS auf.

\subsection{Klinisches Bild der Virus-Pneumonie}

Die HSV-1-Pneumonie tritt spät im Verlauf der Beatmung auf, im Mittel nach 14 Tagen. Es besteht häufig ein Herpes labialis oder eine Gingivo-Stomatitis. Ansonsten ist sowohl bei HSV-1 als auch bei CMV das klinische Bild unspezifisch (Luyt et al. 2011). 


\section{Keine Erreger der nosokomialen Pneumonie}

Diese bedürfen der besonderen Erwähnung, da in der Praxis immer wieder entsprechende Isolate mangels Erwägung anderer Differentialdiagnosen als Erreger der nosokomialen Pneumonie angesehen und entsprechend behandelt werden. Eine solche Praxis bringt jedoch keinen Vorteil für den Patienten, führt vielmehr zu einem erhöhten Selektionsdruck, geht mit einer bedenklichen Toxizität einher und führt zu vermeidbaren Kosten. Neuere Daten belegen sogar eine Assoziation von antimikrobieller (Über-)Therapie und Exzess-Letalität (Kett et al. 2013) bzw. einen prognostisch relevanten Effekt einer ABSgesteuerten Führung der antimikrobiellen Therapie (Schuts et al. 2016).

Alpha-hämolysierende bzw. viridans Streptokokken, Neisseria spp. und Corynebacterium spp. sind Kolonisationskeime der Mundhöhle und sicher keine Erreger einer Pneumonie. Koagulasenegative Staphylokokken sind ebenfalls Kolonisationskeime. Obwohl in einigen Untersuchungen Enterokokken als Erreger einer Pneumonie aufgeführt werden, gibt es bisher keinen Beleg dafür, dass es sich tatsächlich um solche handelt; dies gilt natürlich auch für Vancomycin-resistente Enterokokken (VRE). Schließlich wurde bereits aufgeführt, dass Candida spp. Mikroorganismen sind, die typischerweise durch eine breite antimikrobielle Therapie selektiert werden, aber nicht eigenständige Erreger einer Pneumonie sind.

Merke Dass keine anderen als diese Erreger gefunden wird, belegt gerade nicht, dass diese Isolate Erreger sind und antimikrobiell behandelt werden müssen. Eine solche Situation ist vielmehr ein starker Hinweis darauf, dass keine infektiöse Pneumonie, sondern vielmehr (in vielen Fällen) ein diffuser Alveolarschaden (DAD) vorliegt.

\section{Weiterführende Literatur}

Risikofaktoren für nosokomiale Pneumonien durch S. aureus (MSSA):

Rello J, Quintana E, Ausina V, Puzo C, Net A, Prats G (1990) Risk factors for Staphylococcus aureus nosocomial pneumonia in critically ill patients. Am Rev Respir Dis 142:1320-1324

Prädiktion der S. aureus Pneumonie (MSSA bzw. MRSA) durch Kolonisation der Nase bzw. der Atemwege:

Bergmanns Eur J Clin Microbiol Infect Dis. 1996. 15:437-445

Bergmans D, Bonten M, Gaillard C, de Leeuw P, van Tiel F, Stobberingh E, van der Geest S (1996) Clinical spectrum of ventilator-associated pneumonia caused by methicillin-sensitive Staphylococcus aureus. Eur J Clin Microbiol Infect Dis 15:437-445

Robicsek A, Suseno M, Beaumont JL, Thomson RB Jr, Peterson LR (2008) Prediction of methicillinresistant Staphylococcus aureus involvement in disease sites by concomitant nasal sampling. J Clin Microbiol 46:588-592

Sarikonda KV, Micek ST, Doherty JA, Reichley RM, Warren D, Kollef MH (2010) Methicillinresistant Staphylococcus aureus nasal colonization is a poor predictor of intensive care unit-acquired methicillin-resistant Staphylococcus aureus infections requiring antibiotic treatment. Crit Care Med 38:1991-1995

Rocha LA, Marques Ribas R, da Costa Darini AL, Gontijo Filho PP (2013) Relationship between nasal colonization and ventilator-associated pneumonia and the role of the environment in transmission of Staphylococcus aureus in intensive care units. Am J Infect Control 41:1236-1240

Dangerfield B, Chung A, Webb B, Seville MT (2014) Predictive value of methicillin-resistant Staphylococcus aureus (MRSA) nasal swab PCR assay for MRSA pneumonia. Antimicrob Agents Chemother 58:859-864 
Jang HC, Choi OJ, Kim GS, Jang MO, Kang SJ, Jung SI, Shin JH, Chun BJ, Park KH (2014) Active surveillance of the trachea or throat for MRSA is more sensitive than nasal surveillance and a better predictor of MRSA infections among patients in intensive care. PLoS One. 9(6):e99192

Bedeutung der $\alpha$-Hemolysin-Aktivität für die Entwicklung einer VAP durch MSSA:

Stulik L, Malafa S, Hudcova J, Rouha H, Henics BZ, Craven DE, Sonnevend AM, Nagy E (2014) $\alpha$-Hemolysin activity of methicillin-susceptible Staphylococcus aureus predicts ventilator-associated pneumonia. Am J Respir Crit Care Med 190:1139-1148

MRSA ist nicht sicher mit einer Exzess-Letalität assoziert:

Combes A, Luyt CE, Fagon JY, Wollf M, Trouillet JL, Gibert C, Chastre J; PNEUMA Trial Group (2004) Impact of methicillin resistance on outcome of Staphylococcus aureus ventilator-associated pneumonia. Am J Respir Crit Care Med 170:786-792

Risikofaktoren für eine nosokomiale Pneumonie durch P. aeruginosa:

Celis R, Torres A, Gatell JM, Almela M, Rodríguez-Roisin R, Agustí-Vidal A (1988) Nosocomial pneumonia. A multivariate analysis of risk and prognosis. Chest 93:318-324

Rello J, Ausina V, Ricart M, Puzo C, Quintana E, Net A, Prats G (1994) Risk factors for infection by Pseudomonas aeruginosa in patients with ventilator-associated pneumonia. Intensive Care Med 20:193-198

Talon D, Mulin B, Rouget C, Bailly P, Thouverez M, Viel JF (1998) Risks and routes for ventilatorassociated pneumonia with Pseudomonas aeruginosa. Am J Respir Crit Care Med 157:978-984

Klinisches Bild der VAP durch P. aeruginosa:

Crouch Brewer S, Wunderink RG, Jones CB, Leeper KV Jr (1996) Ventilator-associated pneumonia due to Pseudomonas aeruginosa. Chest 109:1019-1029

Rello J, Mariscal D, March F, Jubert P, Sanchez F, Valles J, Coll P (1998) Recurrent Pseudomonas aeruginosa pneumonia in ventilated patients: relapse or reinfection? Am J Respir Crit Care Med 157:912-916

Risikofaktoren für und Klinik der VAP durch Acinetobacter baumannii:

Lortholary O, Fagon JY, Hoi AB, Slama MA, Pierre J, Giral P, Rosenzweig R, Gutmann L, Safar M, Acar J (1995) Nosocomial acquisition of multiresistant Acinetobacter baumannii: risk factors and prognosis. Clin Infect Dis 20:790-796

Fagon JY, Chastre J, Domart Y, Trouillet JL, Gibert C (1996) Mortality due to ventilator-associated pneumonia or colonization with Pseudomonas or Acinetobacter species: assessment by quantitative culture of samples obtained by a protected specimen brush. Clin Infect Dis 23:538-542

Stenotrophomonas maltophilia: nur selten ein Erreger. Auch die Übertragungswege sind noch nicht abschließend geklärt:

Looney WJ, Narita M, Mühlemann K (2009) Stenotrophomonas maltophilia: an emerging opportunist human pathogen. Lancet Infect Dis 9:312-323

Kataoka D, Fujiwara H, Kawakami T, Tanaka Y, Tanimoto A, Ikawa S, Tanaka Y (2003) The indirect pathogenicity of Stenotrophomonas maltophilia. Int J Antimicrob Agents 22:601-606

Gherardi G, Creti R, Pompilio A, Di Bonaventura G (2015) An overview of various typing methods for clinical epidemiology of the emerging pathogen Stenotrophomonas maltophilia. Diagn Microbiol Infect Dis 81:219-226

Bedeutung von Legionella spp. als Erreger nosokomialer Infektionen und Umgang mit Ausbrüchen:

Boccia S, Laurenti P, Borella P, Moscato U, Capalbo G, Cambieri A, Amore R, Quaranta G, Boninti F, Orsini M, Branca G, Fadda G, Romano-Spica V, Ricciardi G (2006) Prospective 3-year surveillance for nosocomial and environmental Legionella pneumophila: implications for infection control. Infect Control Hosp Epidemiol 27:459-465 
Bartley PB, Ben Zakour NL, Stanton-Cook M, Muguli R, Prado L, Garnys V, Taylor K, Barnett TC, Pinna G, Robson J, Paterson DL, Walker MJ, Schembri MA, Beatson SA (2016) Hospital-wide eradication of a nosocomial legionella pneumophila serogroup 1 outbreak. Clin Infect Dis 62:273-279

Wichtige Untersuchungen zur Rolle der Anaerobier bei VAP mit widersprüchlichen Ergebnissen. Ihre Bedeutung bei der VAP ist (außer bei Empyemen) wahrscheinlich gering:

Doré P, Robert R, Grollier G, Rouffineau J, Lanquetot H, Charrière JM, Fauchère JL (1996) Incidence of anaerobes in ventilator-associated pneumonia with use of a protected specimen brush. Am J Respir Crit Care Med 153:1292-1298

Robert R, Grollier G, Doré P, Hira M, Ferrand E, Fauchère JL (1999) Nosocomial pneumonia with isolation of anaerobic bacteria in ICU patients: therapeutic considerations and outcome. J Crit Care 14:114-119

Marik PE, Careau P (1999) The role of anaerobes in patients with ventilator-associated pneumonia and aspiration pneumonia. A prospective study. Chest 115:178-183

Robert R, Grollier G, Frat JP, Godet C, Adoun M, Fauchère JL, Doré P (2003) Colonization of lower respiratory tract with anaerobic bacteria in mechanically ventilated patients. Intensive Care Med 29:1062-1068

Candida spp.: kein Erreger der nosokomialen Pneumonie:

Rello J, Esandi ME, Díaz E, Mariscal D, Gallego M, Vallès J (1998) The role of Candida sp isolated from bronchoscopic samples in nonneutropenic patients. Chest 114:146-149

el-Ebiary M, Torres A, Fàbregas N, de la Bellacasa JP, González J, Ramirez J, del Baño D, Hernández C, Jiménez de Anta MT (1997) Significance of the isolation of Candida species from respiratory samples in critically ill, non-neutropenic patients. An immediate postmortem histologic study. Am J Respir Crit Care Med 156:583-590

Meersseman W, Lagrou K, Spriet I, Maertens J, Verbeken E, Peetermans WE, Van Wijngaerden E (2009) Significance of the isolation of Candida species from airway samples in critically ill patients: a prospective, autopsy study. Intensive Care Med 35:1526-1531

Terraneo S, Ferrer M, Martín-Loeches I, Esperatti M, Di Pasquale M, Giunta V, Rinaudo M, de Rosa F, Li Bassi G, Centanni S, Torres A (2015) Impact of Candida spp. isolation in the respiratory tract in patients with intensive care unit-acquired pneumonia. Clin Microbiol Infect (im Druck)

Haron E, Vartivarian S, Anaissie E, Dekmezian R, Bodey GP (1993) Primary Candida pneumonia. Experience at a large cancer center and review of the literature. Medicine (Baltimore) 72:137-142

Candida spp. und Interaktion mit anderen Erregern:

Azoulay E, Timsit JF, Tafflet M, de Lassence A, Darmon M, Zahar JR, Adrie C, Garrouste-Orgeas M, Cohen Y, Mourvillier B, Schlemmer B; Outcomerea Study Group (2006) Candida colonization of the respiratory tract and subsequent pseudomonas ventilator-associated pneumonia. Chest 129:110-117

Nseir S, Jozefowicz E, Cavestri B, Sendid B, Di Pompeo C, Dewavrin F, Favory R, Roussel-DelvallezM, Durocher A (2007) Impact of antifungal treatment on Candida-Pseudomonas interaction: a preliminary retrospective case-control study. Intensive Care Med 33:137-142

Hamet M, Pavon A, Dalle F, Pechinot A, Prin S, Quenot JP, Charles PE (2012) Candida spp. airway colonization could promote antibiotic-resistant bacteria selection in patients with suspected ventilatorassociated pneumonia. Intensive Care Med 38:1272-1279

Aspergillus spp. als Erreger der VAP; ein seltener, aber wichtiger Erreger:

Hohl TM, Feldmesser M (2007) Aspergillus fumigatus: principles of pathogenesis and host defense. Eukaryot Cell 6:1953-1963

Meersseman W, Vandecasteele SJ, Wilmer A, Verbeken E, Peetermans WE, van Wijngaerden E (2004) Invasive aspergillosis in critically ill patients without malignancy. Am J Respir Crit Care Med $170: 621-625$ 
Vandewoude KH, Blot SI, Depuydt P, Benoit D, Temmerman W, Colardyn F, Vogelaers D (2006) Clinical relevance of Aspergillus isolation from respiratory tract samples in critically ill patients. Crit Care 10:R3

Taccone FS, van den Abeele AM, Bulpa P, Misset B, Meersseman W, Cardoso T, Paiva JA, BlascoNavalpotro M, De Laere E, Dimopoulos G, Rello J, Vogelaers D, Blot SI; AspICU Study Investigators (2015) Epidemiology of invasive aspergillosis in critically ill patients: clinical presentation, underlying conditions, and outcomes. Crit Care 19:7

Ewig S, Tasci S, Müller E. Fungal VAP (2011) Eur Respir Mon 53:122-137

Viren als Erreger der VAP: Herpesviren können Erreger sein; ihre Bedeutung bei VAP ist noch nicht hinreichend geklärt:

Luyt CE, Combes A, Nieszkowska A, Trouillet JL, Chastre J (2011) Viral VAP. Eur Respir Mon 53:113-121

Zusammenhang von antimikrobieller (Über)Therapie udn Exzess-Letalität sowie prognostisch relevanter Effekt einer ABS-gesteuerten Führung der antimikrobiellen Therapie:

Kett DH, Cano E, Quartin AA, Mangino JE, Zervos MJ, Peyrani P, Cely CM, Ford KD, Scerpella EG, Ramirez JA (2011) Improving medicine through pathway assessment of critical therapy of hospitalacquired pneumonia (IMPACT-HAP) investigators. Implementation of guidelines for management of possible multidrug-resistant pneumonia in intensive care: an observational, multicentre cohort study. Lancet Infect Dis 11:181-189

Schuts EC, Hulscher ME, Mouton JW, Verduin CM, Stuart JW, Overdiek HW, van der Linden PD, Natsch S, Hertogh CM, Wolfs TF, Schouten JA, Kullberg BJ, Prins JM (2016) Current evidence on hospital antimicrobial stewardship objectives: a systematic review and meta-analysis. Lancet Infect Dis $16: 847-856$ 